Check for updates

Cite this: RSC Adv., 2019, 9, 7362

Received 8th January 2019

Accepted 4th February 2019

DOI: $10.1039 / c 9 r a 00168 a$

rsc.li/rsc-advances

\section{Multi-shelled ZnO decorated with nitrogen and phosphorus co-doped carbon quantum dots: synthesis and enhanced photodegradation activity of methylene blue in aqueous solutions}

\author{
Shaojia Song, ${ }^{\text {abc }}$ Kun $\mathrm{Wu}^{\text {abcd }}$ Huadong $\mathrm{Wu}^{\text {abc }}{ }^{\text {Jia Guo }}{ }^{\text {abc }}$ and Linfeng Zhang (DD *abce
}

The presence of organic dyes in wastewater has posed a huge threat to aquatic life and human health. In this study, nitrogen and phosphorus co-doped carbon quantum dot (CQD)-decorated multi-shelled ZnO microsphere photocatalysts (NPCQD/ZnO) were obtained via a simple absorption process; ZnO was prepared by calcining carbon microspheres as the sacrificial template. The as-prepared NPCQD/ZnO showed an obvious multi-shelled structure with the nitrogen and phosphorus co-doped CQDs homogeneously attached onto the inner and outer shells of ZnO. According to the UV-Vis DRS results, all the co-doped, single-doped and undoped carbon quantum dots could enhance the efficiency of absorption of visible light and reduce the optical band gap. Furthermore, the PL characterization results showed that the NPCQD/ZnO composites had lowest fluorescence intensity because the decoration of $\mathrm{ZnO}$ with NPCQDs could effectively reduce the recombination rate of photogenerated electron-hole pairs in the ZnO semiconductor photocatalyst. Importantly, $2 \mathrm{~g}-\mathrm{NPCQD} / \mathrm{ZnO}$ composites exhibited higher photodegradation performance towards methylene blue (MB) than pure $\mathrm{ZnO}$ and even the newly reported series of $\mathrm{ZnO}$ catalysts under the same conditions. Moreover, the degradation obeyed the pseudo-first-order and Langmuir-Hinshelwood kinetics models with a reaction constant of $0.0725 \mathrm{~min}^{-1}$, which was 1.05 times that of pure $\mathrm{ZnO}\left(0.0353 \mathrm{~min}^{-1}\right)$. The NPCQD/ZnO composites not only showed good photocatalytic performance, but also had excellent stability since the photocatalytic activity did not significantly decrease after five cycling tests. In addition, compared with single-doped and undoped carbon quantum dots, $\mathrm{N}$ and $\mathrm{P}$ co-doped carbon quantum dots have more significant efficiency for the modification of semiconductor photocatalysts. The present study shows that the CQDdecorated multi-shelled $\mathrm{ZnO}$ can be regarded as an excellent photocatalyst candidate in the field of water treatment. Moreover, this new concept is helpful in the controllable construction of other multishelled metal oxides decorated with co-doped carbon quantum dots with enhanced photocatalytic properties.

\section{Introduction}

In addition to water shortage, water pollution caused by the discharge of organic and toxic pollutants is becoming a challenge for the sustainable development of the world economy and society. ${ }^{1-5}$ In recent years, the photocatalytic route has proven to

${ }^{a}$ Key Laboratory for Green Chemical Process of Ministry of Education, Wuhan Institute of Technology, Wuhan, 430205, PR China. E-mail: lfzhang@wit.edu.cn

${ }^{b}$ Key Laboratory of Novel Reactor and Green Chemical Technology of Hubei Province, Wuhan Institute of Technology, Wuhan 430205, PR China

'School of Chemical Engineering and Pharmacy, Wuhan Institute of Technology, Wuhan 430205, PR China

${ }^{d}$ College of Post and Telecommunications, Wuhan Institute of Technology, Wuhan 430073, PR China

${ }^{e}$ School of Chemical Engineering and Technology, Tianjin University, Tianjin 300072, PR China be a completely green technique that avoids the use of any auxiliary material in the one-step thorough elimination of pollutants under UV-light irradiation..$^{6-9}$ It has been reported that various semiconductor metal oxides, such as $\mathrm{TiO}_{2}, \mathrm{ZnO}, \mathrm{Bi}_{2} \mathrm{O}_{3}$, $\mathrm{BiOBr}, \mathrm{AgBr}$, etc., can serve as ideal photocatalysts due to their properties of low toxicity, high catalytic efficiency and significant stability ${ }^{10-17}$ Among these, zinc oxide (ZnO), as an important ntype semiconductor photocatalyst, has been widely used in wastewater treatment, environmental purification, and bacteriostatic/photocatalytic sterilization because of its advantages of non-toxicity, high electron exchange performance, low cost and easy accessibility. ${ }^{18-23}$ Under the excitation of UV-Vis light, nano-ZnO can degrade toxic pollutants and transform them into small inorganic molecules. A number of toxic organic pollutants, such as 2,4,6-trichlorophenol, ${ }^{24}$ phenol, ${ }^{25}$ and methyl parathion, ${ }^{26}$ and inorganic pollutants ${ }^{27}$ can be effectively removed 
by this method. However, there are several challenges in using nano-ZnO as a photocatalyst: first, the band gap $\left(\mathrm{E}_{\mathrm{g}}=3.2 \mathrm{eV}\right)$ of $\mathrm{ZnO}$ signifies that $\mathrm{ZnO}$ can only respond to ultraviolet light of less than $380 \mathrm{~nm}$ wavelength (3-5\% of sunlight); second, the photoinduced electrons and hole pairs of $\mathrm{ZnO}$ can easily recombine, and therefore, its effective charge efficiency is low; $;^{28,29}$ and third, the photocorrosion of $\mathrm{ZnO}$ is serious, and $\mathrm{ZnO}$ is not resistant to acids and strong alkalis. Therefore, how to improve the visiblelight response, reduce the recombination rate of photoinduced electron-hole pairs and improve the stability are the directions for the modification and applications of $\mathrm{ZnO}$ photocatalyst.

Compared with other nanostructured materials, semiconductor materials with core-shell or even hollow structures have drawn significant attention because of their obvious advantages such as low density and large specific surface area. ${ }^{30,31}$ Among them, a multi-shelled structure is considered to be a special core-shell structure, which has superior performance when compared with a simple single-layer structure. Wang et al. ${ }^{32}$ obtained multi-shelled ZnO hollow spheres by exercising accurate control and found that the prepared samples exhibited higher energy conversion efficiency than the single-shelled $\mathrm{ZnO}$ hollow spheres. This excellent performance might be attributed to the novel structure that had larger surface areas and the ability to reflect and scatter light. Zeng et $a .^{33}$ prepared hollow $\mathrm{ZnO}$ microspheres with different thicknesses of shells using carbon spheres as a template. The UV-Vis DRS tests showed that $\mathrm{ZnO}$ with a multi-shelled structure had better light response than its single-shelled and nanoparticle counterparts. In addition to the experimental results, the theoretical calculations indicated that $\mathrm{ZnO}$ with a multi-shelled structure had a smaller band gap than that with bare spheres. ${ }^{34}$ Moreover, the degradation experiments showed that $\mathrm{ZnO}$ with this novel structure exhibited better photocatalytic activity towards methyl orange.

Carbon quantum dots (CQDs), as a newly found quasi-zerodimensional nanomaterial, have attracted extensive attention due to their wide absorption band, high optical absorption coefficient and chemical stability. Moreover, the unique upconversion luminescence of CQDs can be excited by light with higher wavelengths and lower frequencies, and then, the CQDs emit low-wavelength light with higher frequency. In addition, the CQDs can be used as electron acceptors to partly reduce the recombination rate of photogenerated electron-hole pairs and then enhance the photocatalytic activity when combined with a photocatalytic semiconductor. ${ }^{35}$ In this regard, the CQDs could play an important role in the design of composite semiconductor photocatalysts. Zhang et al. ${ }^{36}$ synthesized CQD/ZnO nanoflower composites by a two-step method and found that the prepared composite exhibited excellent visible-light catalytic activity towards the degradation of rhodamine B. Ding et $a l .{ }^{37}$ synthesized carbon quantum dots (CQDs) attached on the surface of ZnO with irregular, hierarchical pores. The CQD/ $\mathrm{ZnO}$ composites reported in the literature have been synthesized by a facile process in which the $\mathrm{ZnO}$ foam is dispersed in a CQD solution. The results showed that these composites had good visible-light catalytic performance when compared with the pure $\mathrm{ZnO}$ photocatalysts.
In recent years, researchers have made good progress in the development of synthesis methods, optical properties and application prospects of CQDs. In addition to the CQDS combined with other nanomaterials, CQDs doped with some nonmetallic elements (such as $\mathrm{N}, \mathrm{P}, \mathrm{S}, \mathrm{Cl}$, etc.) can exhibit more stable optical properties, higher quantum yields and extremely excellent catalytic performances, as indicated by numerous previously reported studies. ${ }^{35,38} \mathrm{Hu}$ et al. ${ }^{39}$ synthesized $\mathrm{N}$-doped CQDs, Cl-doped CQDs and undoped CQDs by a hydrothermal method. The fluorescence intensity sequences of the prepared samples were as follows: N-doped CQDs > undoped CQDs > Cldoped CQDs, indicating that the $\mathrm{Cl}$ radicals might decrease the recombination of the photogenerated electron-hole pairs. Huang et al. ${ }^{\mathbf{4}}$ prepared NCQD/TiO ${ }_{2}$ and $\mathrm{CQD} / \mathrm{TiO}_{2}$ composites. The UV-Vis diffuse reflection absorption spectra showed that the $\mathrm{NCQD} / \mathrm{TiO}_{2}$ composite had better photocatalytic performances due to the stronger light absorption intensity and narrower optical band gaps when compared with the $\mathrm{CQD} / \mathrm{TiO}_{2}$ composites.

However, to the best of our knowledge, studies reported on the photocatalysts of $\mathrm{CQD} / \mathrm{ZnO}$ composite materials mainly focus on the structure with single-shelled hollow microspheres. Furthermore, the composited CQDs are usually undoped or single element-doped. In addition, there are a few studies on the preparation and investigation of multielement co-doped CQDs decorated-composited on multi-shelled $\mathrm{ZnO}$ composite materials as an effective photocatalyst. Therefore, multi-shelled ZnO materials, $\mathrm{N}$ and $\mathrm{P}$ single-doped and co-doped CQDs, and a series of $\mathrm{CQD} / \mathrm{ZnO}$ composites were facilely obtained in this study. The photocatalytic performances of the composites were studied through the degradation of simulated wastewater with methylene blue dye under daylight. The morphology, structure, and optical properties of the prepared composites have been analyzed, and the possible mechanism for the enhanced photocatalytic performance has been discussed.

\section{Experimental}

\subsection{Synthesis}

Zinc nitrate hexahydrate $\left(\mathrm{Zn}\left(\mathrm{NO}_{3}\right)_{2} \cdot 6 \mathrm{H}_{2} \mathrm{O}\right)$, sucrose, sodium citrate dihydrate, ammonium dihydrogen phosphate (ADP), phosphoric acid, ethylenediamine (EDA) and ethanol were purchased from the National Pharmaceutical Group Chemical Reagent Co., Ltd. All the chemicals were of reagent grade and used as raw materials without further purification.

2.1.1 Synthesis of the multi-shelled ZnO spheres. In this study, carbonaceous saccharide microspheres were used as the sacrificial templates, and zinc nitrate hexahydrate $\left(\mathrm{Zn}\left(\mathrm{NO}_{3}\right)_{2} \cdot 6 \mathrm{H}_{2} \mathrm{O}\right)$ was used as the metal precursor.

The carbon microsphere templates were synthesized through the emulsion polymerization reaction of sugar under hydrothermal conditions. ${ }^{41}$ The typical synthesis process has been described as follows: $130 \mathrm{~g}$ sucrose is dissolved in $250 \mathrm{~mL}$ deionized water under magnetic stirring for $30 \mathrm{~min}$ at room temperature to form a homogeneous transparent solution (concentration: about $1.5 \mathrm{~mol} \mathrm{~L}^{-1}$ ). After this, the mixture is transferred to a $500 \mathrm{~mL}$ Teflon-lined stainless steel autoclave 


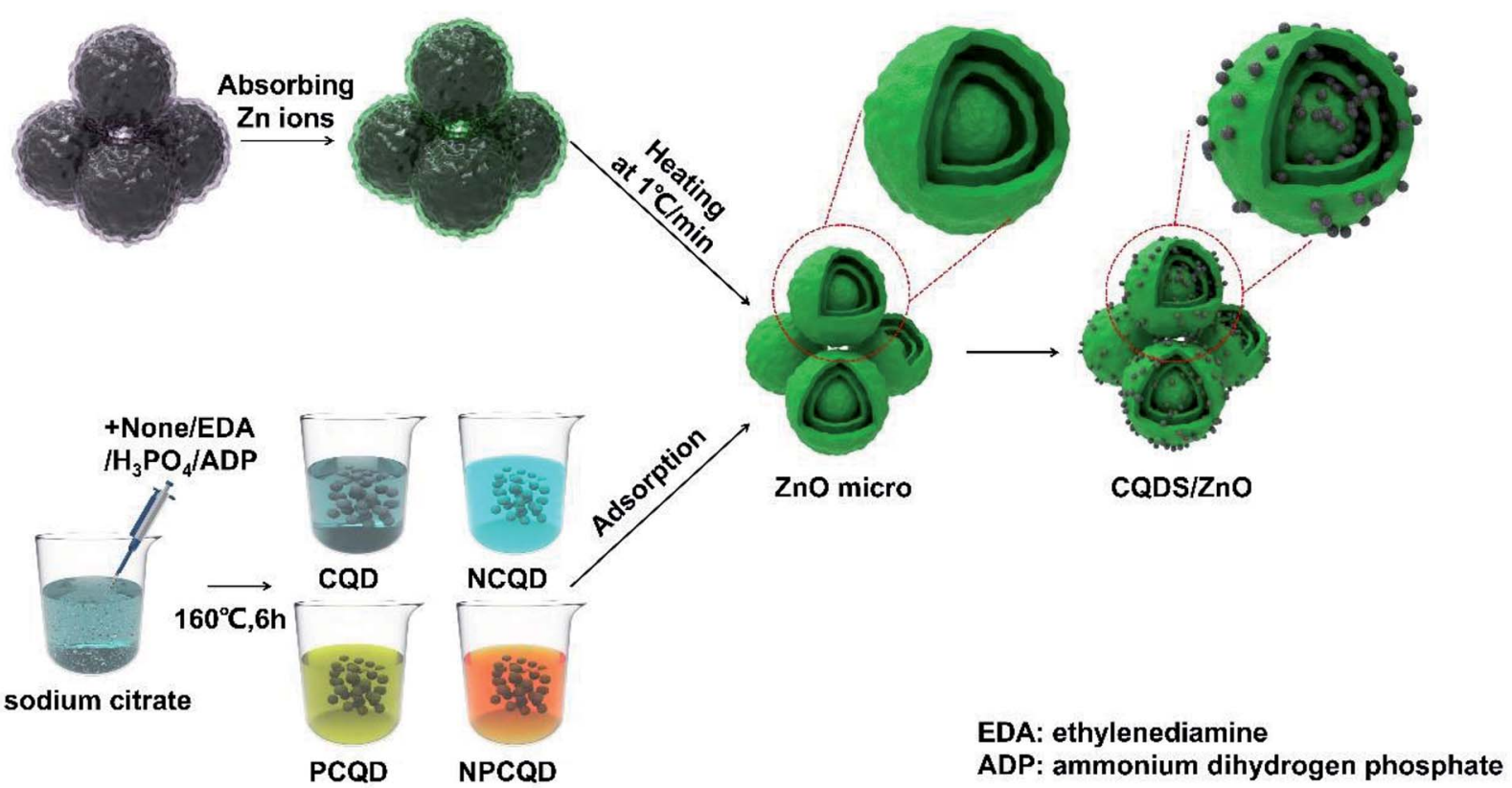

Fig. 1 Schematic of the formation process of the carbon quantum dot-decorated multi-shelled $\mathrm{ZnO}$ composites (CQD/ZnO).

and maintained at $200{ }^{\circ} \mathrm{C}$ for $125 \mathrm{~min}$; then, the autoclave is cooled down to room temperature. The products are alternately washed several times with deionized water and absolute ethanol. Finally, the filter cake is dried at $60^{\circ} \mathrm{C}$ for $12 \mathrm{~h}$ to obtain the carbon microsphere templates.

The preparation of the multi-shelled ZnO spheres was conducted using the method described by Wang et al. ${ }^{32}$ At first, $45 \mathrm{~g}$ zinc nitrate hexahydrate was dissolved in a $30 \mathrm{~mL}$ mixed solution composed of $10 \mathrm{~mL}$ absolute ethanol and $20 \mathrm{~mL}$ deionized water. The mixture was stirred to form a homogeneous solution. Then, $1 \mathrm{~g}$ of carbon microsphere templates was evenly dispersed in the abovementioned solution followed by ultrasonication for $15 \mathrm{~min}$. The resulting suspension was stirred at $40{ }^{\circ} \mathrm{C}$ for $12 \mathrm{~h}$. After this, it was filtered and washed with deionized water. After being dried at $80^{\circ} \mathrm{C}$ for $12 \mathrm{~h}$, the products were heated to $400{ }^{\circ} \mathrm{C}$ at the rate of $1^{\circ} \mathrm{min}^{-1}$, calcined at $400{ }^{\circ} \mathrm{C}$ for $30 \mathrm{~min}$, then heated to $500{ }^{\circ} \mathrm{C}$ at the rate of $1^{\circ} \mathrm{min}^{-1}$ and maintained at this temperature for $60 \mathrm{~min}$ in the muffle furnace to remove the carbon templates. After the furnace was naturally cooled down to room temperature, the resulting product was obtained.

2.1.2 Synthesis of CQDs. The synthesis of carbon quantum dots was carried out according to the synthesis method described by $\mathrm{Xu}$ et $a l .{ }^{42}$ Sodium citrate and ammonium dihydrogen phosphate were used as the carbon and nitrogen and phosphorus sources, respectively. The nitrogen and phosphorus co-doped CQDs were prepared by a hydrothermal method.

The detailed synthesis process has been described herein. For the typical synthesis process, $11.7 \mathrm{~g}$ sodium citrate and $0.5 \mathrm{~g}$ ammonium dihydrogen phosphate (mole ratio is about $10: 1$ ) were dissolved in $40 \mathrm{~mL}$ deionized water. The mixture was constantly stirred for $15 \mathrm{~min}$ at room temperature to form a clear and transparent solution. After this, the mixture was transferred to a $100 \mathrm{~mL}$ Teflon-lined stainless steel autoclave and maintained at $160{ }^{\circ} \mathrm{C}$ for $6 \mathrm{~h}$; then, the autoclave was cooled down to room temperature. The product was filtered by a $0.25 \mu \mathrm{m}$ membrane to remove the impurities and large particles. In addition, the filtrate was further purified by a dialysis membrane $(\mathrm{MWCO}=1000 \mathrm{Da})$ for $24 \mathrm{~h}$ to obtain the final NPCQD solution. The final solution was placed in a refrigerator at $4{ }^{\circ} \mathrm{C}$ for further use.

For comparison, the phosphorus-doped, nitrogen-doped and undoped carbon quantum dots were prepared in this study via similar procedures using different phosphorus and nitrogen sources. For the preparation of the phosphorus-doped carbon quantum dots (PCQDs) and nitrogen-doped carbon quantum dots (NCQDs), ammonium dihydrogen phosphate was replaced with phosphoric acid and ethylenediamine, respectively. The undoped carbon quantum dots (CQDs) were prepared using only sodium citrate without the addition of ammonium dihydrogen phosphate.

2.1.3 Synthesis of the $\mathrm{CQD} / \mathrm{ZnO}$ composites. The preparation of the CQD-decorated multi-shelled ZnO composites was carried out based on the research of Ding et al. ${ }^{37}$ At first, $0.1 \mathrm{~g}$ of the prepared $\mathrm{ZnO}$ was evenly dispersed in $10 \mathrm{~mL}$ deionized water. Then, $0.4 \mathrm{~g}, 0.8 \mathrm{~g}, 1.2 \mathrm{~g}, 2 \mathrm{~g}$, and $2.5 \mathrm{~g}$ NPCQD solutions were added to the abovementioned solution. The mixtures were stirred at room temperature for $12 \mathrm{~h}$. After being centrifuged and washed three times with deionized water, the products were dried at $80{ }^{\circ} \mathrm{C}$ for $12 \mathrm{~h}$ to obtain the final $X \mathrm{~g}$-NPCQD/ZnO composites ( $X=0.4 \mathrm{~g}, 0.8 \mathrm{~g}, 1.2 \mathrm{~g}, 2 \mathrm{~g}$, and $2.5 \mathrm{~g}$ ). For the preparation of $\mathrm{CQD} / \mathrm{ZnO}, \mathrm{NCQD} / \mathrm{ZnO}$, and $\mathrm{PCQD} / \mathrm{ZnO}$, the NPCQD solution was replaced with the CQD solution, NCQD solution and PCQD solution, respectively. The whole synthesis process was shown as in the scheme illustration figure (Fig. 1).

\subsection{Characterization}

The crystal structures of the as-prepared CQD-decorated multishelled ZnO composites were analyzed by X-ray diffraction (XRD D8 ADVANCE and DAVINCI DESIGN) with $\mathrm{Cu}-\mathrm{K} \alpha(\lambda=1.5418 \AA)$ 
radiation in the $2 \theta$ range of $20-80^{\circ}$. The BET surface area, pore volume, and pore size were characterized by nitrogen adsorption at liquid nitrogen temperatures using the Autosorb-iQ instrument (Quantachrome Instruments). The general morphology of the synthesized composites was analyzed by scanning electron microscopy (SEM) using Merlin compact-6178 at the accelerating voltage of $10 \mathrm{kV}$. Transmission electron microscopy (TEM), high-resolution transmission electron microscopy (HRTEM), scanning transmission electron microscopy (STEM) and elemental mapping images were obtained using the Merlin compact-61-78 instrument to further investigate the structure and chemical compositions of the products.

The UV-Vis absorption studies of the composites were conducted using a UV-Vis diffuse reflectance spectrophotometer (U3900 spectrophotometer). The room-temperature photoluminescence (PL) spectrum was obtained using the F-4600 FL spectrophotometer $(5 \mathrm{j} 2400001$ model) at the excitation wavelength of $325 \mathrm{~nm}$.

\subsection{Testing of the photocatalytic activity}

The photocatalytic activity of the catalysts was assessed by the degradation of methylene blue in a double-layer glass reactor. A $300 \mathrm{~W}$ xenon lamp (PLS-SXE300) was used to simulate the daylight source (full working current: $20 \mathrm{~A}$; experimental current: $16 \mathrm{~A}$ ). The typical evaluation process of the catalyst activity has been described as follows. Herein, $20 \mathrm{mg}$ photocatalyst was dispersed in a $200 \mathrm{~mL}$ methylene blue solution (the concentration of MB was $10 \mathrm{mg} \mathrm{L}{ }^{-1}$ ). Before conducting the photocatalytic reaction, the mixed solution was stirred for $30 \mathrm{~min}$ to achieve the adsorptiondesorption equilibrium of the catalyst and MB. Subsequently, the photocatalytic reaction was performed. During the irradiation process, a small amount of dispersion was obtained at $0,5,15,30$, 45, and $60 \mathrm{~min}$ and centrifuged to remove the photocatalysts. Finally, the concentration of MB was measured using a UV-Vis spectrophotometer (PerkinElmer LAMBDA 35) to monitor the changes in the main absorbance centered at $665 \mathrm{~nm}$.

\section{Results and discussion}

\subsection{Structure and morphology of the catalyst}

3.1.1 XRD and $\mathbf{N}_{2}$ adsorption-desorption. Fig. 2 shows the XRD patterns of the prepared photocatalysts. In Fig. 2a, the diffraction peaks located at $2 \theta=31.8^{\circ}, 34.5^{\circ}, 36.3^{\circ}, 56.7^{\circ}, 63.0^{\circ}$, $66.5^{\circ}, 68.1^{\circ}$ and $69.2^{\circ}$ corresponded to the (100), (002), (101), (110), (103), (200), (112) and (201) crystal planes of ZnO (JCPDS: 36-1451). No other diffraction peaks were found. This result indicates that the composites have superb purity of the product. However, no characteristic diffraction peak of carbon is detected. This may be attributed to the small amount (not more than 4\%) and uniform distribution of the carbon quantum dots. ${ }^{37}$ Moreover, it was found that the intensities of the characteristic diffraction peaks for 2 g-CQD/ZnO, 2 g-NCQD/ZnO, 2 g-PCQD/ $\mathrm{ZnO}$, and $2 \mathrm{~g}-\mathrm{NPCQD} / \mathrm{ZnO}$ were significantly reduced as compared to those for pure $\mathrm{ZnO}$. This phenomenon may result from the fact that some of the X-rays are absorbed by the carbon quantum dots loaded on $\mathrm{ZnO}$. In addition, the diffraction peaks for the (101) crystal plane of $2 \mathrm{~g}-\mathrm{CQD} / \mathrm{ZnO}, 2 \mathrm{~g}-\mathrm{NCQD} / \mathrm{ZnO}, 2 \mathrm{~g}$ PCQD/ZnO, and $2 \mathrm{~g}$-NPCQD/ZnO composites are shifted towards a higher angle with varying degrees (Fig. 2b); this may be ascribed to the interaction between $\mathrm{ZnO}$ and the decorated carbon quantum dots (CQDs, NCQDs, PCQDs, and NPCQDs). ${ }^{43}$

The $\mathrm{N}_{2}$ adsorption/desorption isotherm measurements were performed to investigate the specific surface areas and pore size distribution of the NPCQD/ZnO microspheres. The nitrogen adsorption isotherm is a typical IV-type curve with a distinct hysteresis loop in the range of $c a$. 0.4-1.0 $P / P_{0}$ (Fig. 3), indicating the existence of mesoporous and macroporous structures. ${ }^{44}$ The BET surface area is measured to be $17.43 \mathrm{~m}^{2} \mathrm{~g}^{-1}$. In addition, according to the corresponding Barrett-Joyner-Halenda (BJH) pore size distribution curve (inset in Fig. 3), the pore size distribution of the NPCQD/ZnO microspheres is mainly between 5 and $50 \mathrm{~nm}$, which can be mainly attributed to the mesoporous structure of the microsphere surfaces. In addition, the macropores mainly originated from the larger interstitial pores formed by the stacking of the hollow spheres. ${ }^{\mathbf{4 4 , 4 5}}$

3.1.2 SEM and TEM. The SEM images in Fig. 4 show that the photocatalysts are composed of abundant microspheres, which maintain a well-preserved spherical morphology with diameters of about 0.6-0.8 $\mu \mathrm{m}$. A number of broken microspheres were found (as shown in Fig. 4a), which demonstrated the existence of multi-shelled structures. In the scanning TEM (STEM) images shown in Fig. 4c and d, it can be seen that the spheres show an obvious double-shelled structure with particle sizes well consistent with the results observed in the SEM images. More details about the surface of the microsphere can be found in Fig. 4b. Obviously, it was observed that the multishelled ZnO microsphere was composed of many $\mathrm{ZnO}$ nanoparticles. The composites between these nanoparticles are not compact and exhibit some interstitial porosity that can facilitate the inward and outward mass transportation to improve the photocatalytic activity. Fig. 4e-g show the HRTEM images of the $2 \mathrm{~g}$-NPCQD/ZnO composites. The distance of the lattice fringes is determined to be $0.25 \mathrm{~nm}$, which corresponds to the (101) crystallographic spacing plane of $\mathrm{ZnO}$; however, the lattice spacing around $0.31 \mathrm{~nm}$ corresponds to the (002) spacing plane of graphitic carbon, ${ }^{43}$ which is a solid evidence for the formation of the carbon quantum dot-modified $\mathrm{ZnO}$ heterojunction.

The components of the as-prepared 2 g-NPCQD/ZnO composites were further characterized by elemental mapping. Fig. 5b-f show the corresponding elemental distribution of the 2 $\mathrm{g}-\mathrm{NPCQD} / \mathrm{ZnO}$ nanospheres. The elemental mappings of the $2 \mathrm{~g}$ NPCQD/ZnO composites show the presence of $\mathrm{Zn}, \mathrm{O}, \mathrm{C}, \mathrm{N}$ and $\mathrm{P}$, strongly confirming the existence of both phases of $\mathrm{N}$ and P-codoped carbon quantum dots and multi-shelled $\mathrm{ZnO}$; this is in agreement with the results of the HRTEM characterization. In addition, these elements are uniformly distributed inside and outside the surfaces of the $\mathrm{ZnO}$ double-shelled nanospheres. Based on the analysis of the TEM elemental mapping results, it can be concluded that $\mathrm{ZnO}$ and carbon quantum dots are nested within each other, and a complete spherical shell is formed rather than an isolated shell. Fig. $5 \mathrm{~g}$ shows the mass content of the corresponding elements. The content of the $\mathrm{N}$ and P-codoped carbon quantum dots in the composites is about $4 \%$. 

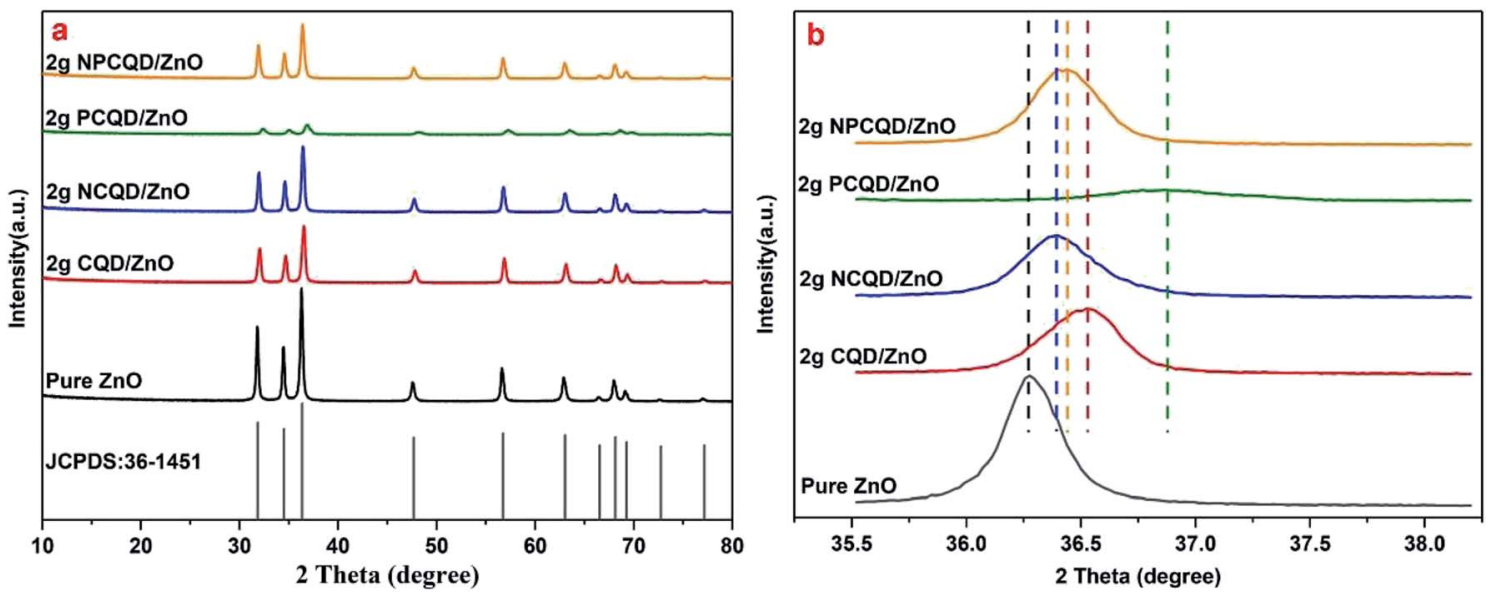

Fig. 2 XRD patterns of the CQD/ZnO composites.

Moreover, carbon, nitrogen and phosphorus can be observed in the inner shell; this proves that the carbon quantum dots can be loaded onto the inner shell of ZnO. It can also be concluded from the TEM elemental mapping results that there are many interstitial pores between $\mathrm{ZnO}$ nanoparticles on the outer shell of the multi-shelled ZnO microspheres; thus, the loaded carbon quantum dots and organic pollutants involved in the photocatalytic reactions can enter the inner space through these holes on the outer shell. This finding is also consistent with the abovementioned SEM images.

\subsection{Photocatalytic activity}

The photocatalytic performances of the carbon quantum dotdecorated $\mathrm{ZnO}$ composites were evaluated for the degradation of $\mathrm{MB}$ (a common pollutant) in water under UV-Vis light irradiation.

Currently, the widely recognized kinetic model for the photocatalytic degradation is the Langmuir-Hinshelwood (LH) model. ${ }^{46,47}$ The corresponding equation is as follows:

$$
r=-\mathrm{d} c / \mathrm{d} t=k K_{\mathrm{c}} /\left(1+K_{\mathrm{c}}\right)
$$

In this equation, $r$ is the total reaction rate of the reactants, $c$ is the concentration of the reactants, $k$ is the reaction rate constant, and $K$ is the apparent adsorption constant. It could be speculated that the adsorption reached saturation because stirring was continued for $30 \mathrm{~min}$ to achieve the adsorptiondesorption equilibrium. Therefore, $K_{\mathrm{c}} \gg 1$, and the eqn (1) can be simplified as follows:

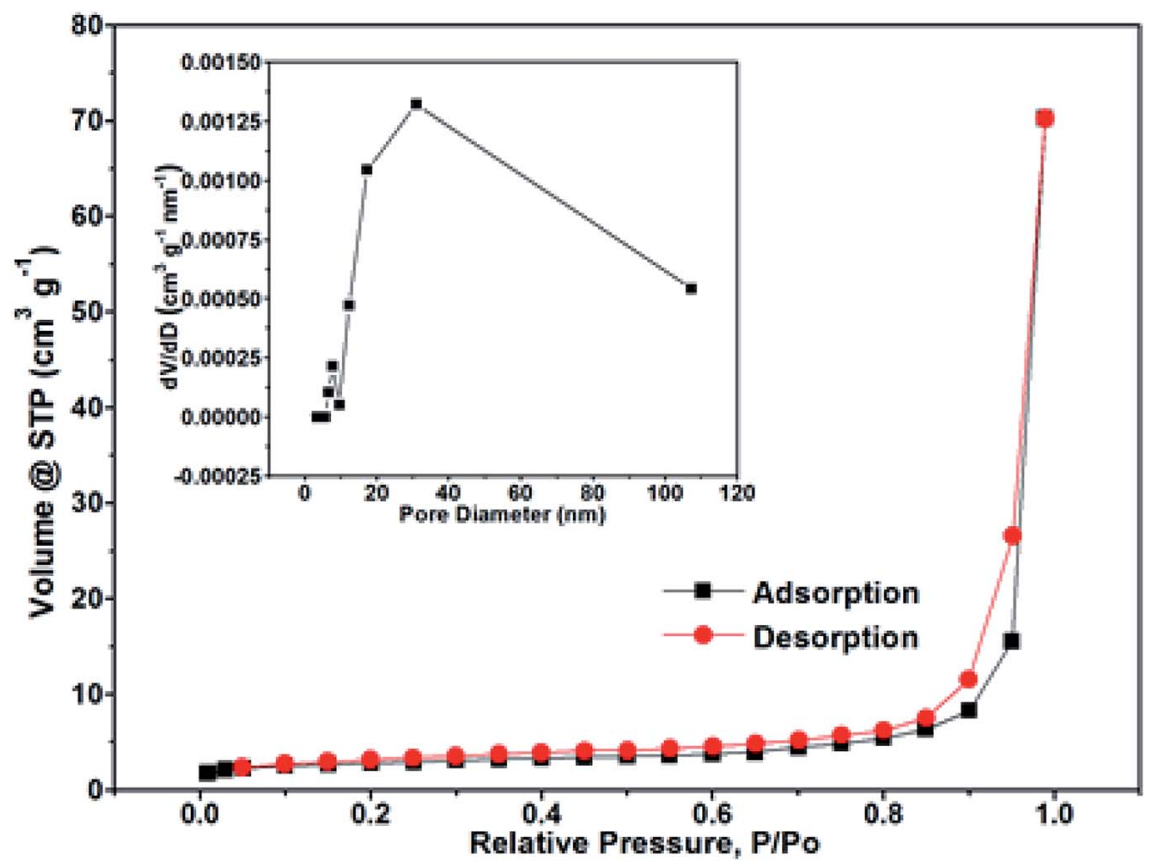

Fig. $3 \mathrm{~N}_{2}$ adsorption-desorption isotherm and pore size distribution (inset) of NPCQD/ZnO. 


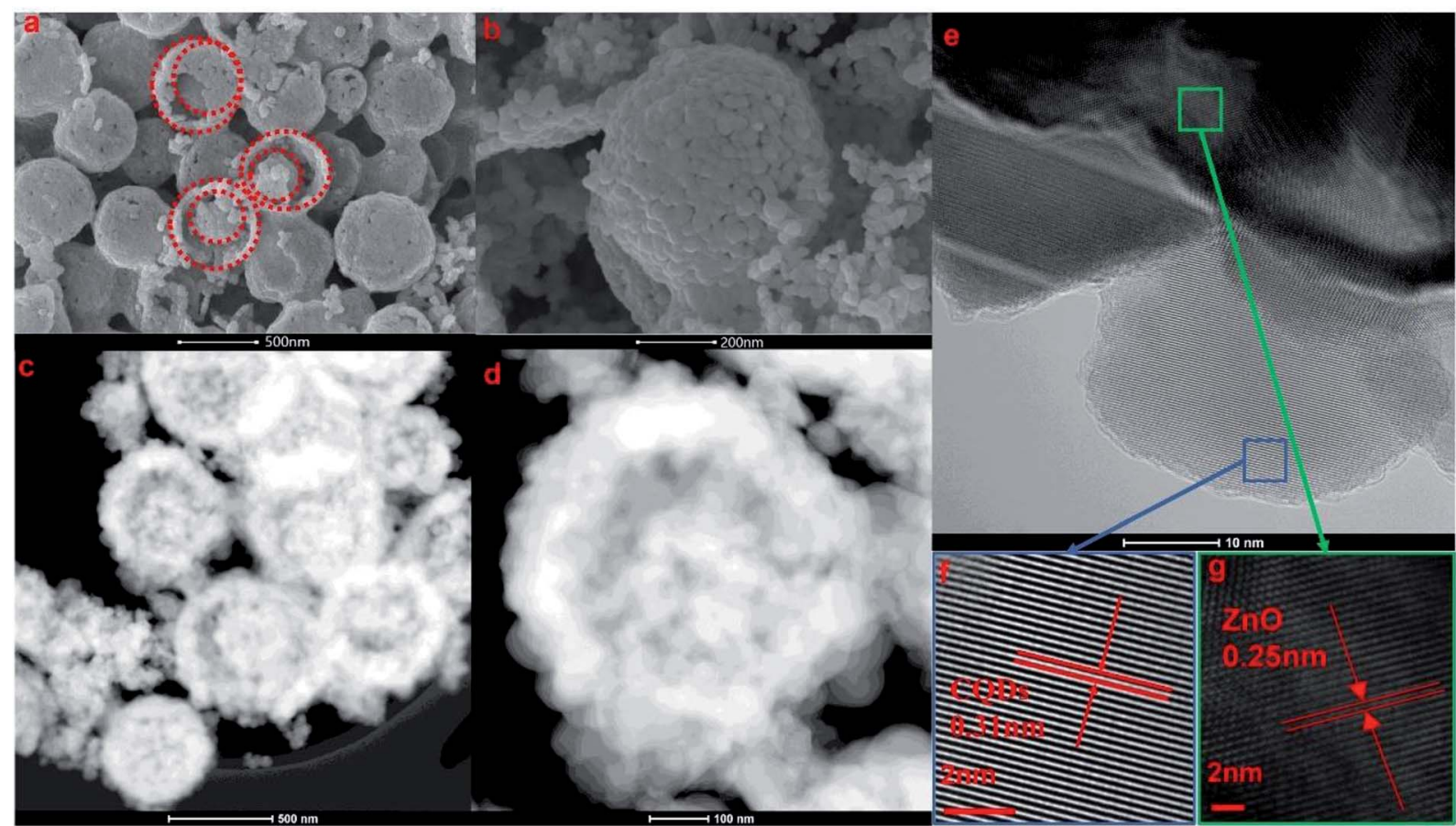

Fig. 4 SEM images ( $a$ and b), scanning TEM images ( $c$ and d), and HRTEM images (e-g) of the prepared 2 g-NPCQD/ZnO composites.

$$
r=-\mathrm{d} c / \mathrm{d} t=k
$$

In conclusion, the reaction rate is independent of $C_{0}$. Linear fitting of the $\ln \left(C_{0} / C_{\mathrm{T}}\right)$-time curve yields a slope that is the reaction rate of the photocatalytic degradation.

Fig. 6 shows the plots of $\ln \left(C_{0} / C_{\mathrm{T}}\right)$ versus irradiation time of the prepared photocatalysts. The $\ln \left(C_{0} / C_{\mathrm{T}}\right)$ versus $t$ presents a good linear relationship, which means that the photodegradation of $\mathrm{MB}$ on these photocatalysts obeys the rules of the LH kinetic model and the first-order reaction kinetics. In
Fig. 6, it is evident that different element-doped carbon quantum dot-decorated $\mathrm{ZnO}$ composites have an appropriate decoration ratio. Interestingly, from the results, we can obviously determine that the best photocatalytic degradation rate for the CQD-decorated multi-shelled $\mathrm{ZnO}$ is at the same decoration ratio (the adsorbent CQD solution is $2 \mathrm{~g}$ ). However, it is evident that the photocatalytic activity of $2.5 \mathrm{~g}$-PCQD/ZnO is worse than that of pure $\mathrm{ZnO}$ (Fig. 6c). This indicates that the composite of multi-shelled $\mathrm{ZnO}$ and a suitable amount of carbon quantum dots can effectively improve the visible
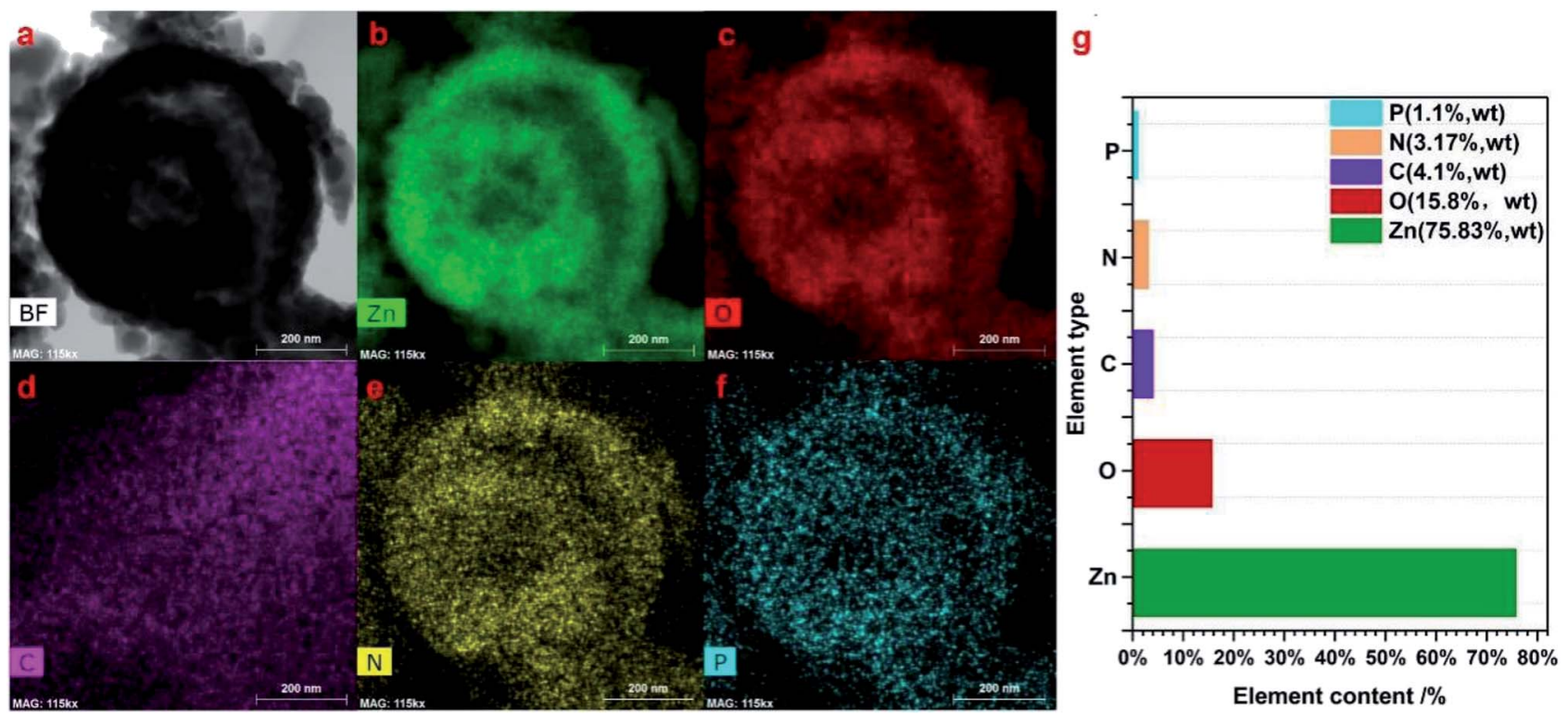

Fig. 5 The corresponding elemental mapping images (a-f) and the elemental distribution of the prepared $2 \mathrm{~g}-\mathrm{NPCQD} / \mathrm{ZnO}$ composites (g). 

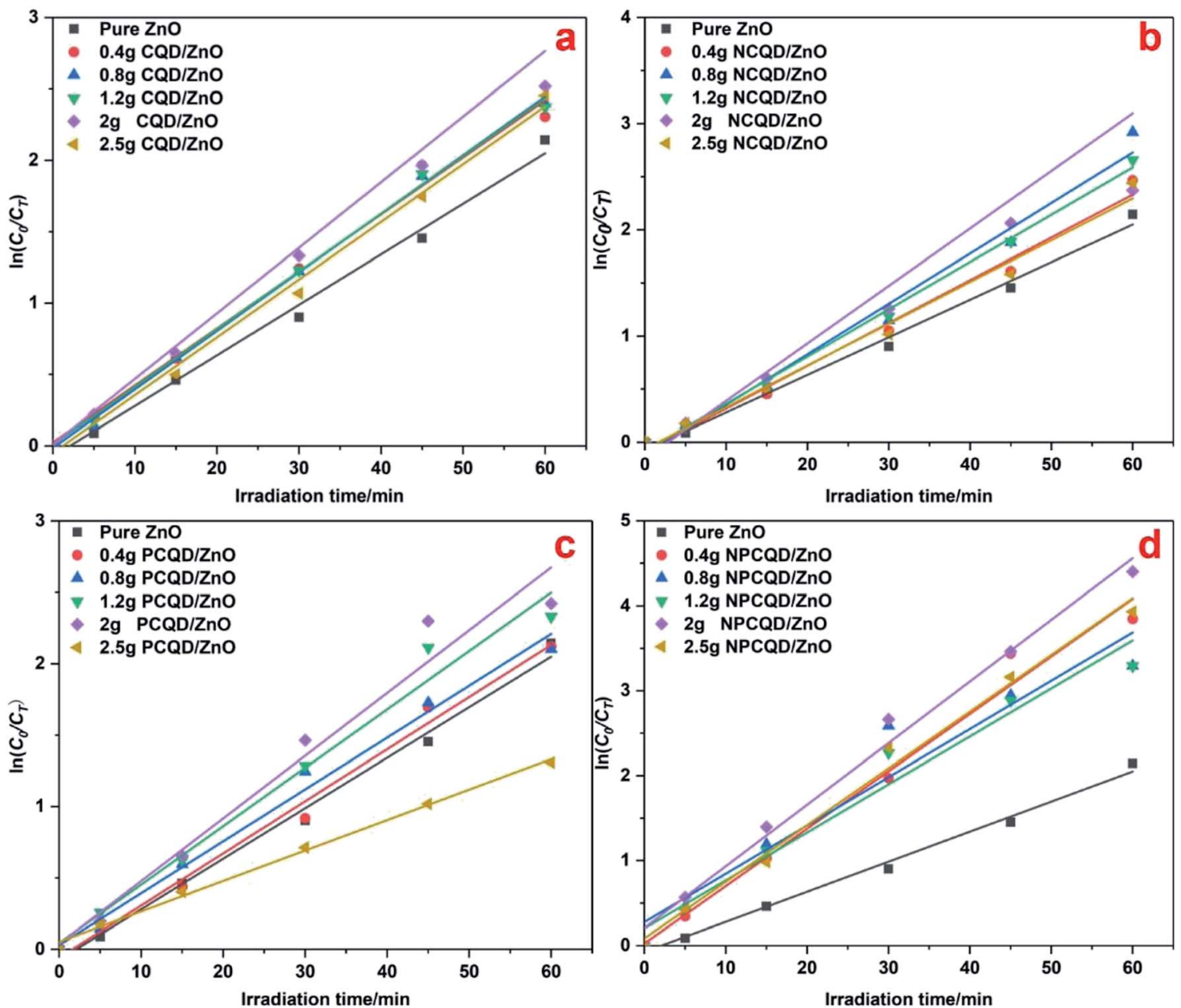

Fig. 6 Photocatalytic degradation kinetic data of the CQD/ZnO composites under UV-Vis light: (a) CQD/ZnO; (b) NCQD/ZnO; (c) PCQD/ZnO; and (d) NPCQD/ZnO.

photocatalytic activity. Therefore, controlling an appropriate amount of the loaded carbon quantum dots is an important factor to improve the photocatalytic activity of the composites.

Among all the composites of CQD-decorated multi-shelled $\mathrm{ZnO}$, the obtained $\mathrm{ZnO}$ composites exhibit highest photocatalytic activity when the amount of the CQD solution is controlled at $2 \mathrm{~g}$. The reason for this may be that the CQD/ZnO composite can improve the light utilization through its unique up-conversion characteristics; ${ }^{35}$ however, the photocatalytic activity of the carbon quantum dots is weaker than that of $\mathrm{ZnO}$. Thus, the higher the content of the carbon quantum dots decorated on the $\mathrm{ZnO}$ shells, the more the competition between the light absorption capacities of $\mathrm{ZnO}$ and carbon quantum dots; this will lead to a decline in the photocatalytic activity; ${ }^{36}$ to study the photocatalytic activity of $\mathrm{ZnO}$ decorated with different kinds of carbon quantum dots, we have selected the same decoration ratio (the amount of CQDs in all the adsorption CQD solutions is controlled at $2 \mathrm{~g}$ ) as a standard to formulate the $C_{\mathrm{T}} /$ $C_{0}$-time and $\ln \left(C_{0} / C_{\mathrm{T}}\right)$-time graphs, as shown in Fig. 7 . It can be clearly seen from Fig. 7 a that the photocatalytic activity of multishelled ZnO has been significantly improved after decoration with the carbon quantum dots. Among these, 2 g-NPCQD/ZnO shows highest photocatalytic activity with a degradation rate of up to $90 \%$ in only 30 min when compared with pure $\mathrm{ZnO}$ (degradation rate is $45 \%$ in $30 \mathrm{~min}$ ). The photocatalytic degradation rate of the photocatalysts follows the order $2 \mathrm{~g}-\mathrm{NPCQD} /$ $\mathrm{ZnO}>2 \mathrm{~g}-\mathrm{PCQD} / \mathrm{ZnO}>2 \mathrm{~g}-\mathrm{CQD} / \mathrm{ZnO}>2 \mathrm{~g}-\mathrm{NCQD} / \mathrm{ZnO}>$ pure ZnO. Fig. 7b exhibits the linear fitting line of the curve of $\ln \left(C_{0} /\right.$ $\left.C_{\mathrm{T}}\right)$ versus time, where the slope represents the photocatalytic reaction rate constant. ${ }^{36}$ Table 1 lists the reaction kinetics equation and the corresponding parameters of the photocatalytic degradation of MB for the as-prepared photocatalysts. The apparent reaction rate constants $(k)$ are $0.0353,0.0424$, $0.0417,0.0439$, and $0.0725 \mathrm{~min}^{-1}$ for pure $\mathrm{ZnO}, 2 \mathrm{~g}$-CQD/ZnO, 2 $\mathrm{g}$-NCQD/ZnO, $2 \mathrm{~g}$-PCQD/ZnO, and $2 \mathrm{~g}$-NPCQD/ZnO, respectively. Thus, the photocatalytic degradation rate of $\mathrm{MB}$ for $2 \mathrm{~g}$ $\mathrm{NPCQD} / \mathrm{ZnO}$ is 1.05 times that for pure ZnO. Furthermore, the $k$ values of $2 \mathrm{~g}$-PCQD/ZnO, $2 \mathrm{~g}-\mathrm{CQD} / \mathrm{ZnO}, 2 \mathrm{~g}-\mathrm{NCQD} / \mathrm{ZnO}$ are about $0.2-0.25$ times that of pure $\mathrm{ZnO}$.

For comparison, Table 2 lists the kinetic data of the degradation of MB by the modified $\mathrm{ZnO}$ photocatalysts reported in the last two years. It can be concluded that $2 \mathrm{~g}$-NPCQD/ZnO has 

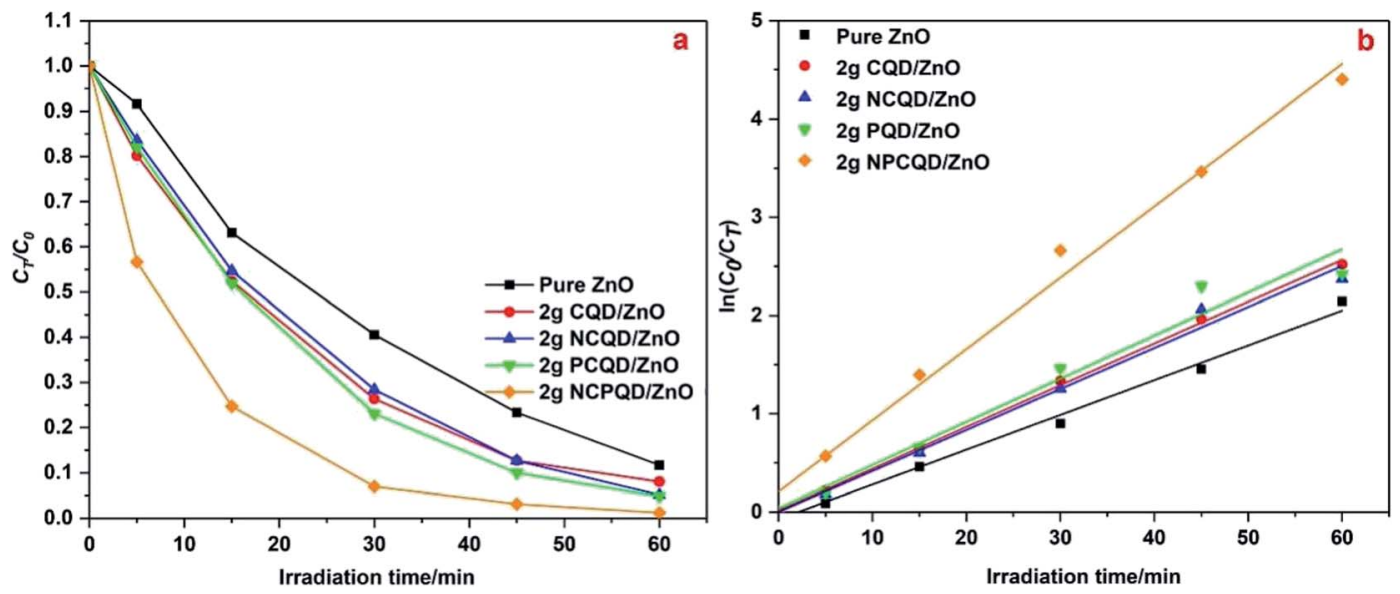

Fig. 7 Photocatalytic degradation of $M B$ with $2 \mathrm{~g}-\mathrm{CQD} / \mathrm{ZnO}$ composites: (a) plots of $C_{T} / C_{0}$ versus time and (b) plots of $\ln \left(C_{0} / C_{T}\right)$ versus time.

Table 1 Kinetic equation and parameters for the degradation of MB by different catalysts

\begin{tabular}{llll}
\hline Catalyst & Fitting linear equation & $\begin{array}{l}\text { Reaction rate } \\
\text { constant }\left(k / \min ^{-1}\right)\end{array}$ & $\begin{array}{l}\text { Linear dependence } \\
\left(R^{2}\right)\end{array}$ \\
\hline Pure ZnO & $\ln \left(C_{0} / C_{\mathrm{T}}\right)=0.0353 t-0.073$ & 0.0353 & 0.99248 \\
2 g-CQDs/ZnO & $\ln \left(C_{0} / C_{\mathrm{T}}\right)=0.0424 t+0.016$ & 0.99887 \\
2 g-NCQDs/ZnO & $\ln \left(C_{0} / C_{\mathrm{T}}\right)=0.0417 t+0.001$ & 0.0424 & 0.98911 \\
2 g-PCQDs/ZnO & $\ln \left(C_{0} / C_{\mathrm{T}}\right)=0.0439 t+0.038$ & 0.0417 & 0.9705 \\
2 g-NPCQDs/ZnO & $\ln \left(C_{0} / C_{\mathrm{T}}\right)=0.07235 t+0.209$ & 0.0439 & 0.98942
\end{tabular}

a competitive photocatalytic degradation activity. Moreover, the stability of the NPCQD/ZnO composite for the degradation of MB was investigated for five cycles (as shown in Fig. 8). It can be seen that the photocatalytic activity is very stable. After the photocatalytic experiment is carried out for five times, the degradation rate efficiency slightly decreases only in the early stages of the photocatalytic reaction. Thus, the as-prepared NPCQD/ZnO composite has excellent chemical stability and can show effective industrial application prospects in the field of water treatment.

\subsection{Mechanism of the photocatalytic activity enhancement}

To reveal the functions of the decorated carbon quantum dots on the multi-shelled ZnO composites, the UV-Vis DRS and PL spectra of these photocatalysts were comprehensively studied.
From the room-temperature UV-Vis DRS results shown in Fig. 9a, it can be observed that after decoration of the carbon quantum dots, the absorption edge of the multi-shelled $\mathrm{ZnO}$ composites shows a small red-shift, and the wavelength for the absorption edge increases. Although the absorption intensity of the carbon quantum dot-decorated multi-shelled $\mathrm{ZnO}$ composites almost remains the same as that of pure $\mathrm{ZnO}$ in the ultraviolet region, the absorbance of the composites in the visible light gradually increases with the decoration of different element-doped carbon quantum dots. In Fig. 9b, it is evident that the curves of $2 \mathrm{~g}$-PCQD/ZnO and $2 \mathrm{~g}$-CQD/ZnO have an intersection. The order of the intensity of absorption in the region larger than $440 \mathrm{~nm}$ changes as follows: $2 \mathrm{~g}-\mathrm{NPCQD} / \mathrm{ZnO}>$ $2 \mathrm{~g}$-CQD/ZnO > $2 \mathrm{~g}$-PCQD/ZnO > $2 \mathrm{~g}$-NCQD/ZnO > pure ZnO. However, the intensity of absorption in the region less than $440 \mathrm{~nm}$ shows the sequence $2 \mathrm{~g}$-NPCQD/ZnO $>2 \mathrm{~g}$-PCQD/ZnO $>$

Table 2 Photocatalytic activities of the CQD/ZnO composites for the degradation of MB

\begin{tabular}{|c|c|c|c|c|c|}
\hline Catalyst & Catalyst dosage & Degraded material & Reaction conditions & $\begin{array}{l}\text { Reaction rate } \\
\text { constant }\left(k / \mathrm{min}^{-1}\right)\end{array}$ & Reference \\
\hline Sn-doped $\mathrm{ZnO}$ & $0.4 \mathrm{~g} \mathrm{~L}^{-1}$ & $0.2 \mathrm{~g} \mathrm{~L}^{-1}$ & $250 \mathrm{~W} \mathrm{Hg}$ lamp & 0.054 & 48 \\
\hline $\mathrm{ZnO} /$ graphene-oxide & - & $0.15 \mathrm{~g} \mathrm{~L}^{-1}$ & $125 \mathrm{~W}$ xenon lamp & 0.040 & 49 \\
\hline $\mathrm{N}-\mathrm{ZnO} / \mathrm{g}-\mathrm{C}_{3} \mathrm{~N}_{4}$ & $1 \mathrm{~g} \mathrm{~L}^{-1}$ & $0.02 \mathrm{~g} \mathrm{~L}^{-1}$ & $300 \mathrm{~W}$ xenon lamp & 0.030 & 50 \\
\hline Tartaric acid/ZnO & $0.8 \mathrm{~g} \mathrm{~L}^{-1}$ & $0.01 \mathrm{~g} \mathrm{~L}^{-1}$ & $300 \mathrm{~W}$ mercury lamp & 0.422 & 51 \\
\hline $\mathrm{AgBr} / \mathrm{g}-\mathrm{C}_{3} \mathrm{~N}_{4} / \mathrm{ZnO}$ & $0.4 \mathrm{~g} \mathrm{~L}^{-1}$ & $0.005 \mathrm{~g} \mathrm{~L}^{-1}$ & $300 \mathrm{~W}$ halogen lamp & 0.041 & 52 \\
\hline $\mathrm{ZnO} / \mathrm{NiFe}_{2} \mathrm{O}_{4}$ & $0.4 \mathrm{~g} \mathrm{~L}^{-1}$ & $0.2 \mathrm{~g} \mathrm{~L}^{-1}$ & $360 \mathrm{~W}$ UV-lamp & 0.029 & 53 \\
\hline $\mathrm{ZnO} / \mathrm{CuO}$ BTC & $1 \mathrm{~g} \mathrm{~L}^{-1}$ & - & Philips TL $15 \mathrm{~W} / 5 \mathrm{BLB}$ & 0.059 & 54 \\
\hline $\mathrm{Fe}, \mathrm{Ni}-\mathrm{ZnO}$ & $1 \mathrm{~g} \mathrm{~L}^{-1}$ & $0.02 \mathrm{~g} \mathrm{~L}^{-1}$ & $300 \mathrm{~W}$ xenon lamp & 0.012 & 55 \\
\hline
\end{tabular}




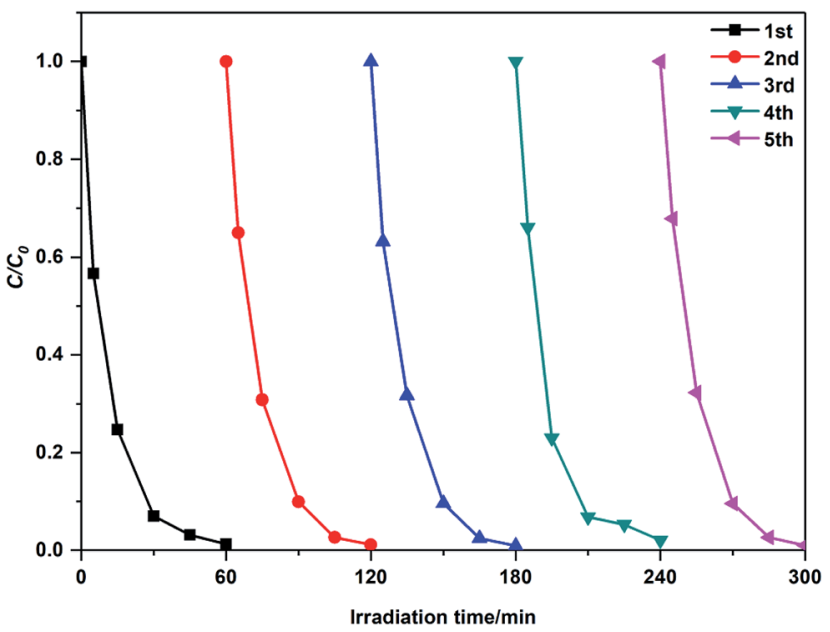

Fig. 8 Cycle activity of 2 g-NPCQDs/ZnO for MB degradation with cycling for five times.

2 g-CQD/ZnO $>2$ g-NCQD/ZnO > pure ZnO. Earlier studies have shown that $\mathrm{ZnO}$ mainly responds to ultraviolet light when used as a photocatalyst. Furthermore, the light source output in the photocatalytic performance is mainly concentrated in the region less than $440 \mathrm{~nm}$. Hence, the order of absorption in the ultraviolet light region is well consistent with the photocatalytic performance.

Fig. 10 shows a plot of $\alpha(h v)^{0.5} v s$. photon energy $(h v)$ for the multi-shelled $\mathrm{ZnO}$ composites decorated with different elementdoped carbon quantum dots. The direct band-gap energy $\left(E_{\mathrm{d}}\right)$ can be defined by the simplified formula $\alpha(h v)=C\left(h v-E_{\mathrm{d}}\right)^{2} \cdot{ }^{34}$ Herein, $\alpha$ is the absorption coefficient, $h v$ is the photon energy with frequency $v$, and $C$ is a constant. Hence, $E_{\mathrm{d}}$ can be calculated by the plot of $\alpha(h v)^{0.5} v s$. photon energy $(h v)$. The calculated direct band-gaps of $2 \mathrm{~g}$-NPCQD/ZnO and $2 \mathrm{~g}$-PCQD/ZnO exhibit the lowest value $(3.13 \mathrm{eV})$, which is lower than those of $2 \mathrm{~g}$-NCQD/ $\mathrm{ZnO}$ and $2 \mathrm{~g}-\mathrm{CQD} / \mathrm{ZnO}(3.14 \mathrm{eV})$ and pure $\mathrm{ZnO}(3.165 \mathrm{eV})$.

The narrower band gap and higher visible-light absorption lead to the participation of more photogenerated electron-hole pairs in the photocatalytic reaction. Thus, the composites of multi-shelled $\mathrm{ZnO}$ and doped carbon quantum dots exhibit promising applications as photocatalysts under visible light as compared to pure multi-shelled ZnO.

Normally, the photocatalytic performance of the catalysts may be influenced by several factors. It is significantly affected by the range of light response and the efficiency of light absorption. Moreover, the recombination of photogenerated electron holes is an important factor.

To confirm the photogenerated charge separation and recombination behaviors of the as-synthesized multi-shelled $\mathrm{ZnO}$ composites, the photoluminescence (PL) spectra of different composites were examined at $325 \mathrm{~nm}$ excitation (Fig. 11). Obviously, all the composites exhibit obvious PL signals with similar curve shapes; this suggests that the decoration of the CQDs does not generate a new PL signal. All the samples show strong visible emission spectra. In the background of this broad spectrum, one obvious emission peak could be observed, namely, the blue emission peak at the center of $475 \mathrm{~nm}$. It is generally believed that blue ray emission resistance of the semiconductor $\mathrm{ZnO}$ is due to the surface oxygen vacancies and defects. ${ }^{56}$ The PL intensities of the single element-doped and undoped CQDs are stronger than that of pure $\mathrm{ZnO}$; this indicates that the composite samples decorated with these carbon quantum dots have more surface defects.

It is clearly observed that the 2 g-NPCQD/ZnO composites show lowest emission intensity. Moreover, the sequence of the PL intensities for the single element-doped and undoped carbon quantum dots is as follows: $2 \mathrm{~g}$-NCQD/ZnO $<2 \mathrm{~g}$-CQD/ $\mathrm{ZnO}<2 \mathrm{~g}$-PCQD/ZnO. The lower PL intensity indicates rapid charge transfer and lower recombination rate of the photogenerated electron-hole pairs, thus improving the photocatalytic activity.

Another confusing question worth considering is why the order of the PL intensity for the carbon quantum dot-decorated $\mathrm{ZnO}$ composites is 2 g-NPCQD/ZnO $<2$ g-PCQD/ZnO $<2$ g-CQD/ $\mathrm{ZnO}<2$ g-NCQD/ZnO. As is well-known, many defects exist on the surface of the carbon quantum dots that can form visible
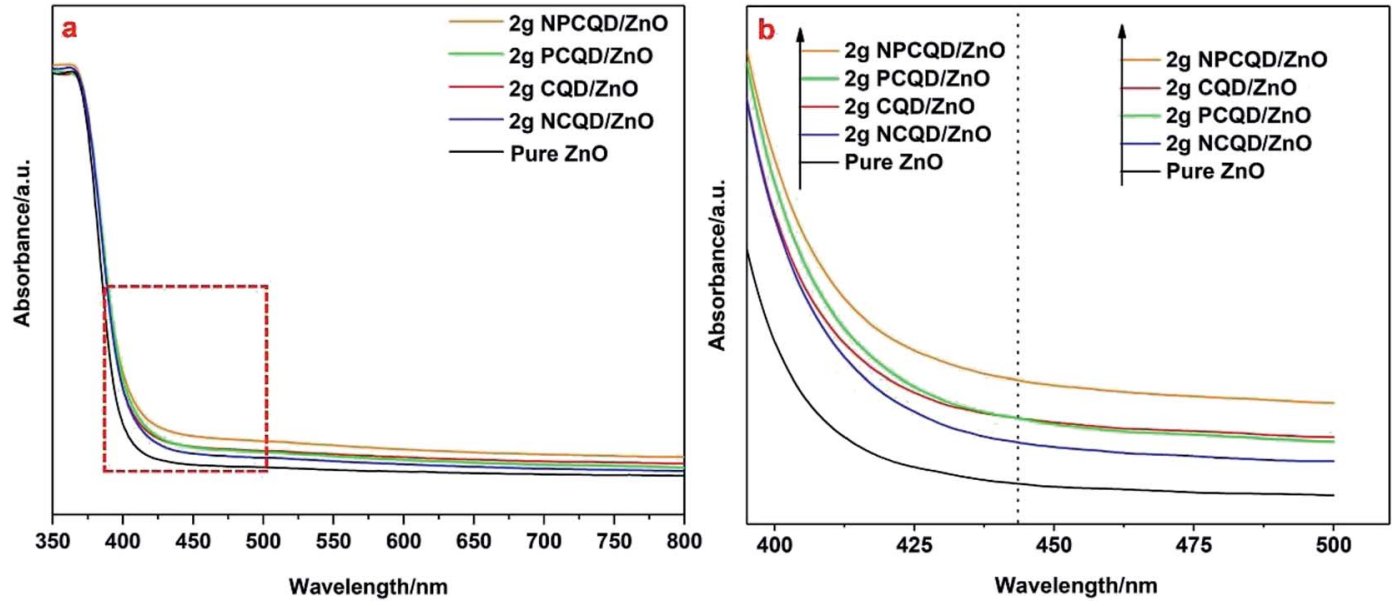

Fig. 9 UV-Vis absorption spectra (a) and band edges of the as-synthesized multi-shelled ZnO composites decorated with different element -doped carbon quantum dots (b). 


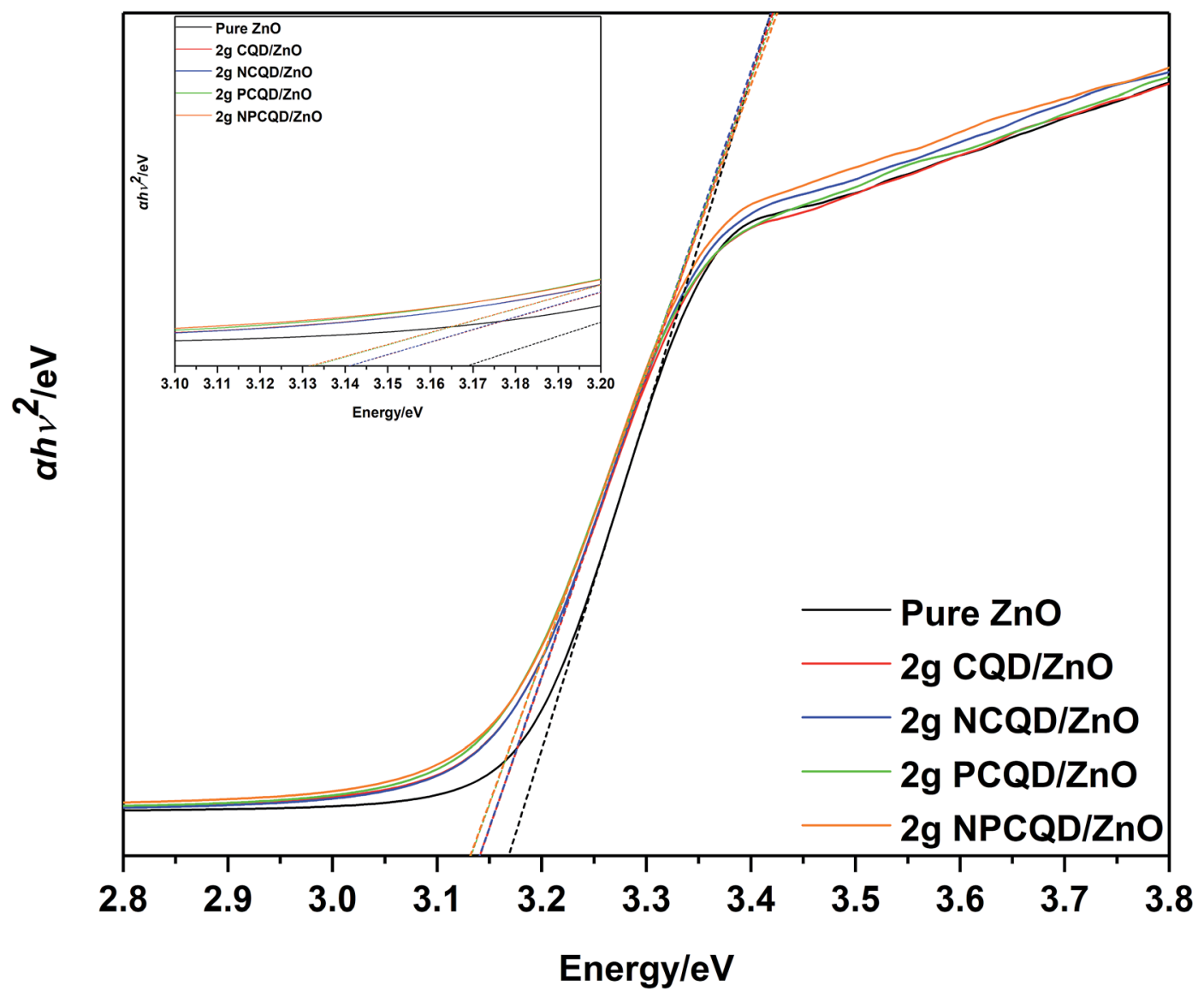

Fig. 10 Tauc plot of the as-synthesized multi-shelled $\mathrm{ZnO}$ composites decorated with different element-doped carbon quantum dots.

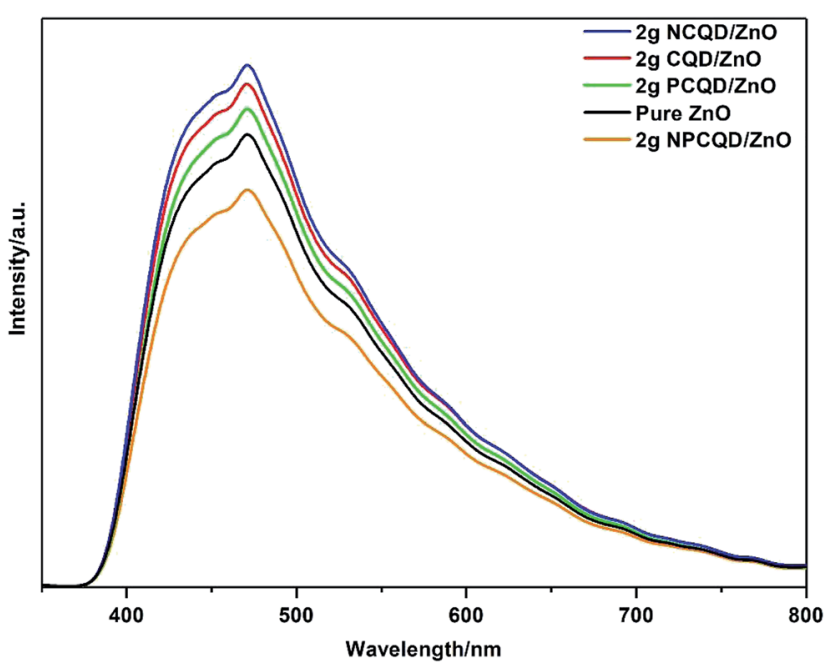

Fig. $11 \mathrm{PL}$ spectra of the as-synthesized multi-shelled $\mathrm{ZnO}$ composites decorated with different element-doped carbon quantum dots.

band gaps, and these band gaps will constantly move from the interior towards the surface; this will result in energy band gap bending. ${ }^{57,58}$ The potential induced by the band gap bending affects the separation efficiency of the electrons and holes. The main cause of the surface band gap bending is the distribution and type of the surface groups. The NCQD/ZnO and PCQD/ZnO composites contain only nitrogen-containing and phosphorouscontaining groups, respectively. All the composites of carbon quantum dots and $\mathrm{ZnO}$ have oxygen-containing groups.

Earlier studies have shown that oxygen-containing and nitrogen-containing groups induce upward band gap bending, and the same band gap bending direction slows down the separation of the electrons and holes. ${ }^{39}$ On the other hand, phosphorous-containing groups may induce a downward band gap bending, and this band gap bending direction is opposite to that in the case of the oxygen-containing or nitrogen-containing groups. Therefore, this band gap bending may be equivalent to the formation of a miniature electric field inside the composites, which may accelerate the separation of electrons from holes. ${ }^{39}$ The surface oxygen vacancies and defects of the $\mathrm{ZnO}$ composites can be increased by loading carbon quantum dots; therefore, these composites can exhibit better visible light response. In addition, due to the concurrence of oxygencontaining, nitrogen-containing and phosphorous-containing groups in the NPCQD/ZnO composites, bandgap bending induced by these three groups forms a miniature electric field in the photocatalysts, which promotes the transfer of photogenerated electron-hole pairs, and thus, the composites show lowest fluorescence intensity. 


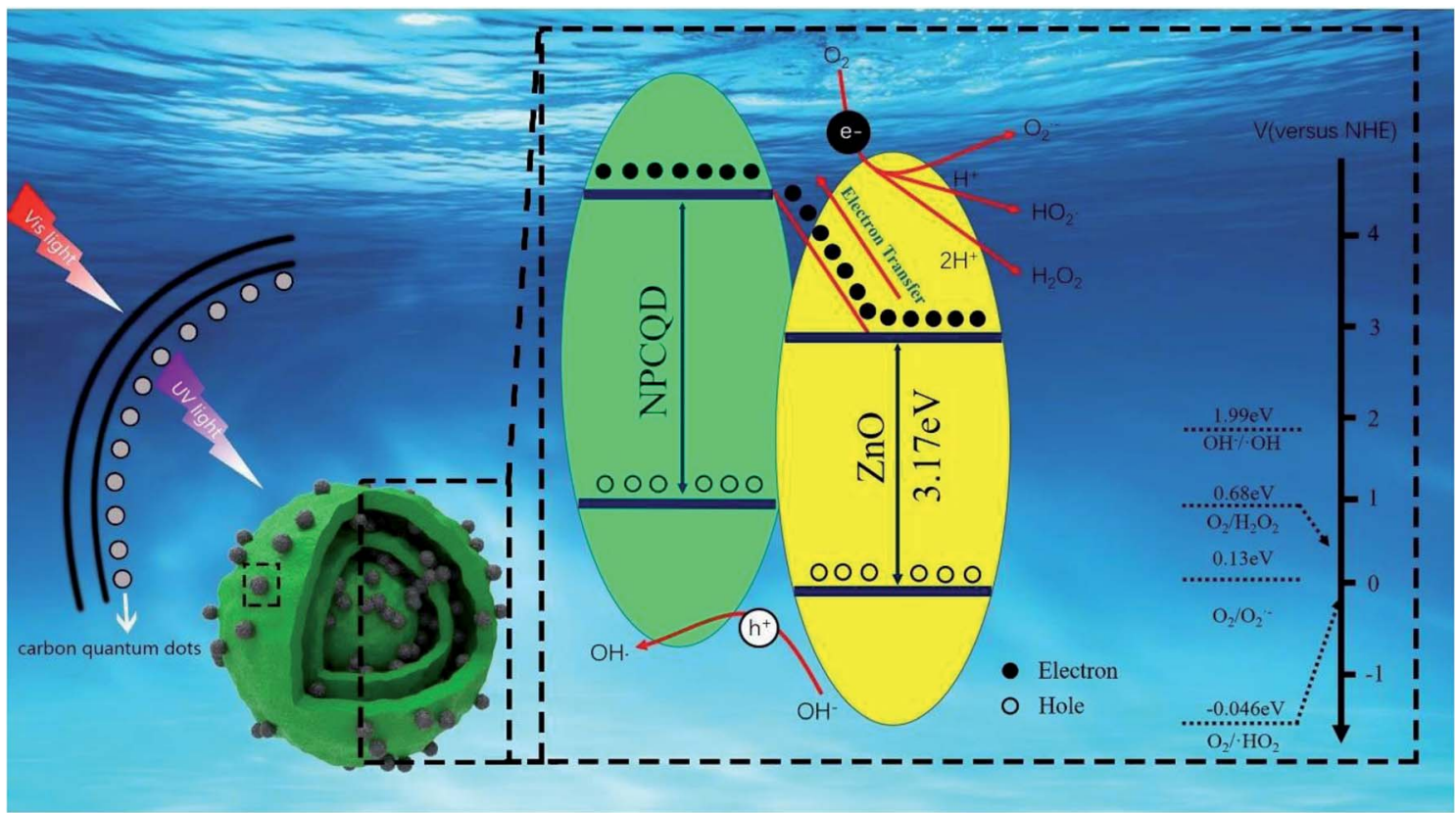

Fig. 12 The proposed schematic diagram for the photocatalytic degradation of MB by the NPCQD/ZnO composites under UV-Vis irradiation.

For the present enhancement analysis, the following mechanism was proposed under UV-Vis irradiation (Fig. 12). As shown in the previous literature, ${ }^{59}$ after CQDs are introduced into multi-shelled $\mathrm{ZnO}$ to form a composite system, a certain amount of visible light is converted into shorter-wavelength irradiation energy, which improves the light response range and absorption intensity. As a result, more photogenerated electron hole pairs are produced to participate in the photocatalytic reaction.

According to the previous studies, the LUMO level of the carbon quantum dots is in the range of $4.2-4.4 \mathrm{eV}{ }^{40}$ and the conduction band (CB) of $\mathrm{ZnO}$ is $2.86 \mathrm{eV}$. Therefore, it is thermodynamically favorable to transfer electrons from the $\mathrm{CB}$ of ZnO to the VB of carbon quantum dots. Therefore, NPCQDs can promote the separation of photogenerated electron-hole pairs; this is in agreement with the PL results. On the other hand, when photocatalysts are irradiated, fewer photons enter the crystal structure. Some of these photons are directly absorbed, and the others are reflected. Due to the multi-shelled structure, more photons remain within the $\mathrm{ZnO}$ microsphere structure until completely absorbed; this is also considered as one of the reasons for the efficient photocatalysis. Based on the abovementioned advantages, the composites of N, P-co-doped carbon quantum dots and multi-shelled $\mathrm{ZnO}$ show efficient degradation rate for the MB solution and may be regarded as an excellent photocatalyst candidate in the field of water treatment.

\section{Conclusion}

In this study, a series of multi-shelled $\mathrm{ZnO}$ composites decorated with different element-doped/undoped carbon quantum dots were fabricated via a simple electrostatic adsorption approach. Among them, carbon quantum dots doped with different elements (mainly nitrogen and phosphorus) were synthesized by adjusting the reactant precursors under the same hydrothermal conditions. Multi-shelled $\mathrm{ZnO}$ was obtained by calcining the carbon microspheres as the sacrificial template. After characterization, the results showed that all the carbon quantum dots were attached onto the inner and outer shells of $\mathrm{ZnO}$ with uniform dispersion. The photocatalytic performances of these catalysts were evaluated for the degradation of MB under UV-Vis light irradiation. The pseudo-firstorder reaction constant for the degradation of $\mathrm{MB}$ by the NPCQD/ZnO composite was two times that of pure ZnO. Moreover, the UV-Vis DRS spectrum indicates that NPCQD/ZnO has a wider light response range, higher light absorption intensity and smaller optical band gap than pure $\mathrm{ZnO}$ that result in more photogenerated electron-hole pairs. In addition, the PL spectrum revealed that the decoration of NPCQDs allowed better separation of the photogenerated electron-hole pairs on the ZnO surface. In addition, single-doped nitrogen and phosphorus CQDs together with undoped CQDs exhibited improvement in the light response range, light absorption intensity and optical band gap. In addition, compared with single-doped and undoped carbon quantum dots, N, P co-doped carbon quantum dots have more significant efficiency with respect to the modification of semiconductor photocatalysts. The present study shows that the prepared carbon quantum dot-decorated multi-shelled $\mathrm{ZnO}$ composites can be regarded as an excellent photocatalyst candidate in the field of water treatment. Furthermore, this new concept is helpful in the controllable construction of other multi-shelled metal oxides decorated with co-doped carbon quantum dots with enhanced properties for photocatalytic activity. 


\section{Conflicts of interest}

There are no conflicts to declare.

\section{Acknowledgements}

This project was financially supported by the projects of China Postdoctoral Science Foundation funded project (2017M610491), the Key program of Natural Science Foundation of Hubei Province (No. 2016CFA079), and the Scientific Research Plan Project of Education Department of Hubei Province (No. B2017057).

\section{References}

1 Z. Wen, J. Ke, J. Xu, S. Guo, Y. Zhang and R. Chen, Chem. Eng. J., 2018, 343, 416-426.

2 S. Luo, F. Qin, Y. Ming, H. Zhao, Y. Liu and R. Chen, J. Hazard. Mater., 2017, 340, 253-262.

3 R. Wang, G. Cheng, Z. Dai, J. Ding, Y. Liu and R. Chen, Chem. Eng. J., 2017, 327, 371-386.

4 J. Ding, Z. Dai, F. Qin, H. Zhao, S. Zhao and R. Chen, Appl. Catal., B, 2017, 205, 281-291.

5 J. Ke, M. Adnan Younis, Y. Kong, H. Zhou, J. Liu, L. Lei and Y. Hou, Nano-Micro Lett., 2018, 10, 69.

6 H. Tang, Z. Dai, X. Xie, Z. Wen and R. Chen, Chem. Eng. J., 2019, 356, 472-482.

7 H. Yang, X. Hu, C. Su, Y. Liu and R. Chen, Phys. Chem. Chem. Phys., 2017, 19, 31666-31674.

8 J. Ke, X. Duan, S. Luo, H. Zhang, H. Sun, J. Liu, M. Tade and S. Wang, Chem. Eng. J., 2017, 313, 1447-1453.

9 F. Xu, G. Cheng, S. Song, Y. Wei and R. Chen, ACS Sustainable Chem. Eng., 2016, 4, 7013-7022.

10 Y. Gan, Y. Wei, J. Xiong and G. Cheng, Chem. Eng. J., 2018, 349, 1-16.

11 H. Long, L. Bao, A. A. Habeeb and P. Lu, Opt. Quantum Electron., 2017, 49, 1-8.

12 J. Ding, Z. Dai, F. Tian, B. Zhou, B. Zhao, H. Zhao, Z. Chen, Y. Liu and R. Chen, J. Mater. Chem. A, 2017, 5, 23453-23459.

13 M. Yuan, F. Tian, G. Li, H. Zhao, Y. Liu and R. Chen, Ind. Eng. Chem. Res., 2017, 56, 5935-5943.

14 Z. Dai, F. Qin, H. Zhao, J. Ding, Y. Liu and R. Chen, ACS Catal., 2016, 6, 3180-3192.

15 Y. Tu, Q. Fu, X. Niu, J. Sang, Z. Tan and X. Zou, J. Mater. Sci. Technol., 2013, 29, 1053-1058.

16 Z. Dai, F. Qin, H. Zhao, F. Tian, Y. Liu and R. Chen, Nanoscale, 2015, 7, 11991-11999.

17 H. Liu, M. Luo, J. Hu, T. Zhou, R. Chen and J. Li, Appl. Catal., $B$, 2013, 140-141, 141-150.

18 H. Yang, Q. Zhang, Y. Chen, Y. Huang, F. Yang and Z. Lu, Carbohydr. Polym., 2018, 201, 162-171.

19 Z. Gao, K. Sasaki and X. Qiu, Langmuir, 2018, 34, 5386-5395.

20 J. Sun, P. Cai, F. Pan, L. Zhang, Z. Liu, Z. Liu, Y. Cao and J. Chen, ACS Appl. Mater. Interfaces, 2018, 10, 11094-11100.

21 S. Wang, C. Huang, L. Pan, Y. Chen, X. Zhang, F. Aleem and J. Zou, Catal. Today, 2018, DOI: 10.1016/j.cattod.2018.10.059.
22 L. Pan, T. Muhammad, L. Ma, Z. Huang, S. Wang, L. Wang, J. Zou and X. Zhang, Appl. Catal., B, 2016, 189, 181-191.

23 L. Pan, S. Wang, W. Mi, J. Song, J. Zou, L. Wang and X. Zhang, Nano Energy, 2014, 9, 71-79.

24 R. Xu, J. Cui, R. Tang, F. Li and B. Zhang, Chem. Eng. J., 2017, 326, 647-655.

25 X. Feng, H. Guo, K. Patel, H. Zhou and X. Lou, Chem. Eng. J., 2014, 244, 327-334.

26 E. Evgenidou, I. Konstantinou, K. Fytianos, I. Poulios and T. Albanis, Catal. Today, 2007, 124, 156-162.

27 H. Wang, L. Liang, X. Cheng, Y. Luo and S. Sun, Photochem. Photobiol., 2017, 94, 17-26.

28 Z. Lu, J. Gao, Q. He, J. Wu, D. Liang, H. Yang and R. Chen, Carbohydr. Polym., 2017, 156, 460-469.

29 S. Wang, J. Wu, H. Yang, X. Liu, Q. Huang and Z. Lu, J. Mater. Sci.: Mater. Med., 2017, 28, 1-8.

30 J. Xiong, G. Cheng, F. Qin, R. Wang, H. Sun and R. Chen, Chem. Eng. J., 2013, 220, 228-236.

31 Q. Li, H. Li, R. Wang, G. Li, H. Yang and R. Chen, J. Alloys Compd., 2013, 567, 1-9.

32 Z. Dong, X. Lai, J. E. Halpert, N. Yang, L. Yi, J. Zhai, D. Wang, Z. Tang and L. Jiang, Adv. Mater., 2012, 24, 1046-1049.

33 X. Zeng, J. Yang, L. Shi, L. Li and M. Gao, Nanoscale Res. Lett., 2014, 9, 1-10.

$34 \mathrm{~V}$. Editor and M. Balkanski, Optical properties of solids, North-Holland Pub. Co., American Elsevier, 1980.

35 S. Y. Lim, W. Shen and Z. Gao, Chem. Soc. Rev., 2015, 44, 362381.

36 X. Zhang, J. Pan, C. Zhu, Y. Sheng, Z. Yan, Y. Wang and B. Feng, J. Mater. Sci.: Mater. Electron., 2015, 26, 2861-2866.

37 D. Ding, W. Lan, Z. Yang, X. Zhao, Y. Chen, J. Wang, X. Zhang, Y. Zhang, Q. Su and E. Xie, Mater. Sci. Semicond. Process., 2016, 47, 25-31.

38 Y. Wang, Y. Zhao, F. Zhang, L. Chen, Y. Yang and X. Liu, New J. Chem., 2016, 40, 8710-8716.

39 S. Hu, R. Tian, Y. Dong, J. Yang, J. Liu and Q. Chang, Nanoscale, 2013, 5, 11665-11671.

40 Y. Q. Zhang, D. K. Ma, Y. G. Zhang, W. Chen and S. M. Huang, Nano Energy, 2013, 2, 545-552.

41 Q. Wang, H. Li, L. Chen and X. Huang, Carbon, 2001, 39, 2211-2214.

42 Q. Xu, B. Li, Y. Ye, W. Cai, W. Li, C. Yang, Y. Chen, M. Xu, N. Li, X. Zheng, J. Street, Y. Luo and L. Cai, Nano Res., 2018, 11, 3691-3701.

43 S. Muthulingam, K. Bin Bae, R. Khan, I. H. Lee and P. Uthirakumar, RSC Adv., 2015, 5, 46247-46251.

44 J. Feng, H. Guo, S. Wang, Y. Zhao and X. Ma, Chem. Eng. J., 2017, 321, 401-411.

45 Y. Han, X. Dong, C. Zhang and S. Liu, J. Power Sources, 2012, 211, 92-96.

46 J. Mu, C. Shao, Z. Guo, Z. Zhang, M. Zhang, P. Zhang, B. Chen and Y. Liu, ACS Appl. Mater. Interfaces, 2011, 3, 590-596.

47 X. Zou, Y. Dong, S. Li, J. Ke and Y. Cui, Sol. Energy, 2018, 169, 392-400.

48 J. Lin, Z. Luo, J. Liu and P. Li, Mater. Sci. Semicond. Process., 2018, 87, 24-31. 
49 P. Kumbhakar, A. Pramanik, S. Biswas, A. K. Kole, R. Sarkar and P. Kumbhakar, J. Hazard. Mater., 2018, 360, 193-203.

50 Y. Liu, H. Liu, H. Zhou, T. Li and L. Zhang, Appl. Surf. Sci., 2019, 466, 133-140.

51 S. Duo, L. Zhang, R. Zhong, Z. Liu, L. Huang, T. Liu and Y. Zhang, J. Alloys Compd., 2018, 768, 214-229.

52 E. Boorboor Azimi, A. Badiei and M. Hossaini Sadr, J. Phys. Chem. Solids, 2018, 122, 174-183.

53 J. T. Adeleke, T. Theivasanthi, M. Thiruppathi, M. Swaminathan, T. Akomolafe and A. B. Alabi, Appl. Surf. Sci., 2018, 455, 195-200.
54 D. Tuncel and A. N. Ökte, Catal. Today, 2018, DOI: 10.1016/ j.cattod.2018.10.049.

55 D. Neena, K. K. Kondamareddy, H. Bin, D. Lu, P. Kumar, R. K. Dwivedi, V. O. Pelenovich, X. Z. Zhao, W. Gao and D. Fu, Sci. Rep., 2018, 8, 1-12.

56 A. Khan, W. M. Jadwisienczak and M. E. Kordesch, Phys. E, 2006, 33, 331-335.

57 Z. Zhang and J. T. Yates, Chem. Rev., 2012, 112, 5520-5551.

58 J. Chen, D. F. Ollis, W. H. Rulkens and H. Bruning, Water Res., 1999, 33, 1173-1180.

59 J. Ke, X. Li, Q. Zhao, B. Liu, S. Liu and S. Wang, J. Colloid Interface Sci., 2017, 496, 425-433. 